\begin{tabular}{l|l} 
REVISTA & $\begin{array}{l}\text { Revista Educación } \\
\text { ISSN: 0379-7082 } \\
\text { ISSN: 2215-2644 } \\
\text { revedu@ gmail.com } \\
\text { Universidad de Costa Rica } \\
\text { Costa Rica }\end{array}$
\end{tabular}

\title{
Perspectivas éticas de la docencia universitaria en América Latina: retos y desafíos en el siglo XXI
}

Baltodano Enríquez, Manuel

Perspectivas éticas de la docencia universitaria en América Latina: retos y desafíos en el siglo XXI

Revista Educación, vol. 44, núm. 2, 2020

Universidad de Costa Rica, Costa Rica

Disponible en: http://www.redalyc.org/articulo.oa?id=44062184018

DOI: https://doi.org/10.15517/revedu.v44i2.38677

Esta obra está bajo una Licencia Creative Commons Atribución-NoComercial-SinDerivar 3.0 Internacional. 


\title{
Perspectivas éticas de la docencia universitaria en América Latina: retos y desafíos en el siglo XXI
}

\author{
Ethical Perspectives of Teaching at Latin American Universities: Challenges in the 21st century
}

Manuel Baltodano Enríquez

Ministerio de Educación Pública, Costa Rica

manuelinsidehome@gmail.com

(iD http://orcid.org/0000-0003-2599-2192

\author{
DOI: https://doi.org/10.15517/revedu.v44i2.38677 \\ Redalyc: http://www.redalyc.org/articulo.oa?id=44062184018
}

Recepción: 23 Septiembre 2019

Aprobación: 05 Mayo 2020

\section{Resumen:}

Este ensayo plantea algunas reflexiones en torno a los principios éticos que subyacen al ejercicio de la docencia universitaria. En este sentido, la universidad pública latinoamericana enfrenta una serie de retos y desafíos éticos, ante la voraz globalización y políticas económicas las cuales intentan convertirla en una empresa que responda a intereses neoliberales, mediante la mercantilización de bienes y servicios educativos financieramente rentables. En atención a la problemática expuesta, la universidad, como institución milenaria, ha transitado a lo largo del tiempo por diversas transformaciones; desde la docencia, la investigación y la acción social. No obstante, desde finales del siglo pasado, las instituciones de educación superior en todo el mundo se enfrentan a la necesidad de orientar su acción hacia la venta de servicio y la internacionalización, lo cual plantea un debate ético en relación con la misión de la universidad pública y su incidencia en el quehacer docente, los principios que rigen el desarrollo del currículo universitario para la formación integral del estudiantado y la responsabilidad social que la universidad pública latinoamericana posee debido a la complejidad de las problemáticas sociales y las desigualdades que ha traído consigo el modelo económico capitalista, imperante en la mayoría de los países del mundo.

Palabras clave: Ética, Enseñanza superior, Desarrollo de la educación, Formación profesional, Responsabilidad social.

\section{Abstract:}

This article reflects on the underlying ethical principles associated with teaching at the college/university level. Latin America 's public universities currently face ethical challenges amidst voracious globalization and economic policies which threaten to turn them into companies that respond to neoliberal interests by commercializing educational commodities and services that are financially profitabile. In light of these circumstances, universities, which have existed for the past thousand years, have undergone various transformations ranging from a focus on teaching to research and social action. Nevertheless, since the end of the last century, institutions of higher learning worldwide, have been faced with the need to focus on selling services and become more international, which poses an ethical debate regarding the mission of public universities and its impact on the professional endeavours of the faculty and the principles that govern university curricululm development. An effort must be made not only to provide a well-rounded education for students, but one that also embraces social responsitlity, intrinsically associated to Latin American state universities, given the complex social struture in the region that has wrough on an economic capitalist model, as is the dominant structure in the majority of countries throughout the world.

KEYwORDS: Ethics, Higher Education, Educational Development; Professional Training, Social Responsibility.

\section{INTRODUCCIÓN}

En el contexto mundial, el ejercicio de la docencia universitaria cobra especial importancia desde la perspectiva ética para la formación integral del estudiantado y la responsabilidad social de la universidad pública; respondiendo con ello a los principios más arraigados en la misión histórica de la universidad; la docencia, la investigación y la acción social. Desde esta posición las perspectivas éticas desde el ejercicio de la docencia universitaria se entienden como el conjunto de deberes normativos en el ejercicio de su profesión 
que asume la persona docente de manera consciente, libre y voluntaria para generar valor al quehacer educativo contribuyendo así con el desarrollo humano y social en general (Bunge, 2005; Sánchez, 1974).

Por otro lado, la persona docente debería estar éticamente comprometida con la misión y la visión que orientan el quehacer de la universidad pública, pues el ejercicio de la docencia universitaria implica una alto compromiso social y vocación por el desarrollo humano de las personas estudiantes. Asimismo, desde el contexto universitario latinoamericano es necesario que el profesorado forje una actitud que permita asumir con compromiso y coherencia el ejercicio de la profesión en aras de favorecer el desarrollo humano y el progreso social. Por otra parte, es necesario establecer mecanismos de evaluación que hagan tangibles los resultados que se desprenden de los proyectos de investigación y extensión liderados por el profesorado, que repercuten en el desarrollo humano del estudiado y el bienestar de la sociedad en general.

El inicio del nuevo milenio ha traído consigo profundas trasformaciones de la sociedad en las cuales intervienen factores económicos, políticos y sociales, donde la acelerada expansión y desarrollo de las tecnologías digitales, la globalización y el modelo económico capitalista, han marcado las pautas en cuanto al establecimiento de nuevas relaciones entre las instituciones, los individuos, y la sociedad en general, mediante nuevos acuerdos y tendencias para satisfacer las necesidades planetarias y corporativas (Gavrila, 2018; Predazzi, 2012; Scott, 2006). En relación con lo anterior, la universidad pública latinoamericana se ha visto obligada a ejecutar ajustes en su misión, objetivos estratégicos y metas con el propósito de satisfacer las diversas demandas.

Considerando lo antes expuesto, en la posmodernidad las instituciones de educación superior en América Latina se han visto amenazadas primordialmente por políticas neoliberales de mejora de la calidad, que fomentan solapadamente mecanismos colonizadores que atentan contra la docencia, la investigación y la acción social, misión que históricamente ha tenido; precarizando el ejercicio de la docencia en la universidad (D' Antoni, 2013; Scott, 2006). En este caso, cabe mencionar que la condiciones actuales caracterizan el ejercicio de la profesión docente universitaria son establecidas a partir de las características del contexto económico y político neoliberal, lo cual ha provocado una baja sustancial en la calidad de la docencia universitaria y la masificación de productos educativos que muchas veces no contribuyen con la mejora de las problemáticas sociales y el desarrollo humano de las personas estudiantes (D’ Antoni, 2013). Por ello, a nivel latinoamericano debe existir un consenso colectivo en la opinión pública que ha fomentado la pérdida de referentes creíbles y la incertidumbre valorativa para hacer ver ante la sociedad a la universidad pública como una institución poco rentable y como trinchera para favorecer privilegios de algunos grupos que ostentan el poder dentro de esta. De lo antes descrito, se plantean en la Figura 1 cuatro desafíos éticos que se deben asumir desde el ejercicio de la docencia universitaria para preservar la misión de la universidad pública como referente científico, social y humano, a saber: 


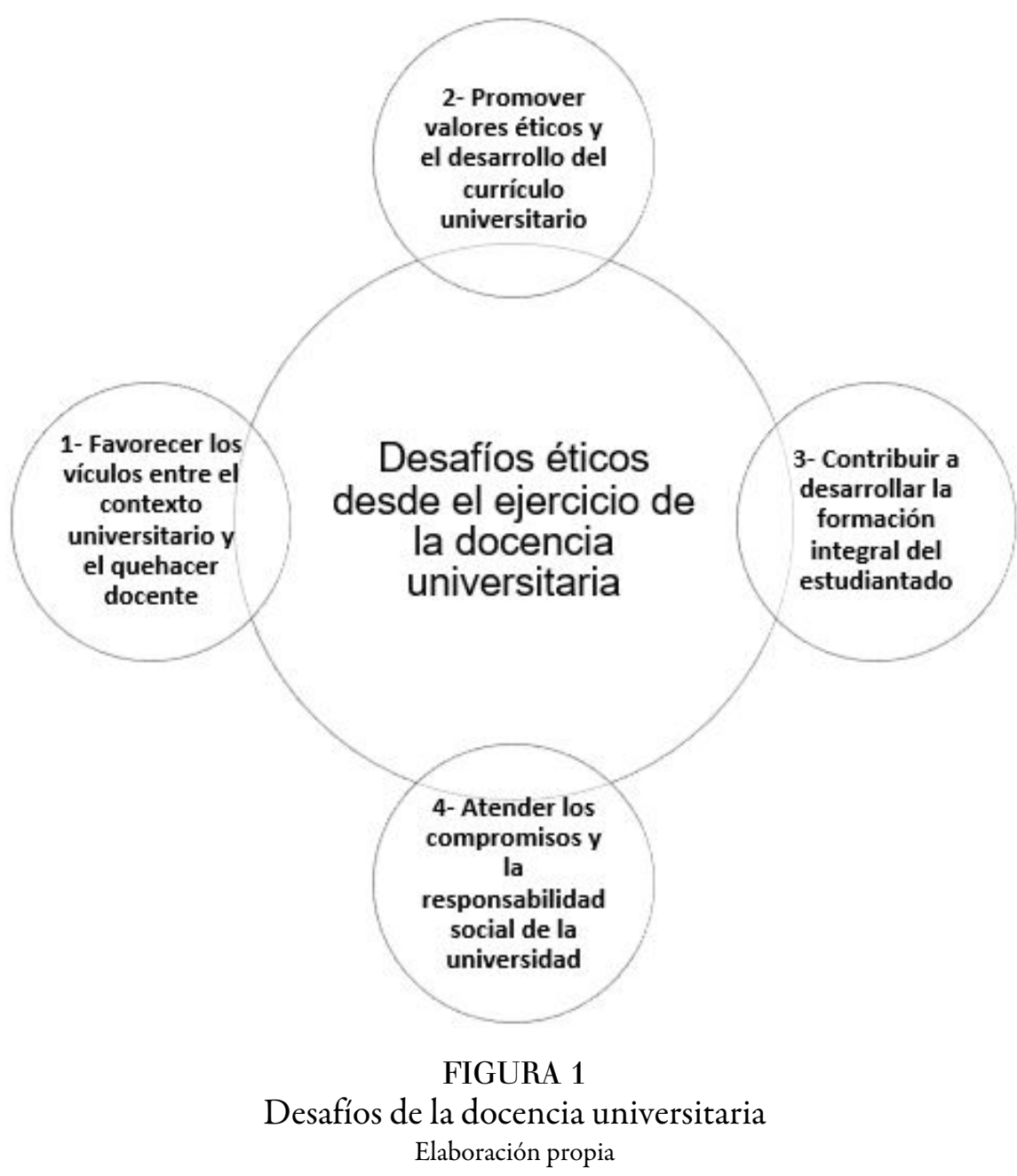

En relación con el contexto universitario y su vínculo con el quehacer docente, la universidad pública latinoamericana debería dialogar con los grupos sociales para coadyuvar a la atención de problemas complejos que implican la formación de personas con compromiso social y humano, atendiendo a la vez las necesidades del profesorado. Por ello, para la comunidad universitaria es preciso contar con condiciones óptimas de infraestructura universitaria, una excelente mediación pedagógica y sobre todo experiencias innovadoras más allá del salón de clases (Carvajal, 2013).

En el mismo orden de las ideas anteriores, en la docencia universitaria los valores éticos y el currículo universitario conlleva al planteamiento reflexivo sobre el para qué se enseña y aprende, y cuál es la intensión educativa de las políticas e ideologías detrás de la formación de las personas. Igualmente, el currículo como proyecto formativo que responde a las demandas de la sociedad, las tendencias científicas y las necesidades humanas, conducen a una formación integral del ser humano que trascienda la simple trasmisión de conocimientos y que conduzca a una genuina discusión sobre la importancia que tiene dicha formación en la vida de las personas.

Por otra parte, la formación integral en la universidad como práctica cotidiana puede llevarse a cabo de muchas formas, sin embargo, dicha formación implica el replanteamiento de los programas de estudio con el fin de enriquecer los cocimientos actitudinales y el sentido crítico del ser humano frente a los conocimientos disciplinares y hacia la vida misma. En relación con lo anterior, vale la pena que desde la universidad pública se enriquezcan las mallas curriculares con el fin de ofrecer asignaturas que conlleven a la autocrítica y reflexión sobre el accionar del ser humano respecto a las problemáticas personales y sociales. 
Desde el ámbito de la responsabilidad social, la universidad pública latinoamericana como organización podría promover políticas éticas que de una u otra manera coadyuven con el desarrollo social y que promueven la solución de diversas problemáticas. No obstante, el proceso que conduce al planteamiento de iniciativas comunales universitarias ha de ser gestionado y planificado rigurosamente, de tal forma que se puedan hacer diagnósticos comunitarios que permitan un trabajo efectivo y coherente con la misión y proyección social de la universidad (Vallaeys, 2016). Es por lo que se requiere para la comunidad universitaria en general la investigación, la planificación detallada de las propuestas o proyectos, trasparencia, sentido social y redes de apoyo participativas donde el estudiantado y el profesorado tomen la iniciativa para emprender trabajos comunales con alto valor social, que afiancen el compromiso que la universidad tiene con consigo misma y con los sectores sociales menos favorecidos por la acción universitaria. Por último, el propósito general del ensayo es discutir y generar perspectivas sobre los siguientes ejes temáticos: el contexto universitario y el quehacer docente, la ética en el desarrollo de currículo universitario, la formación integral del estudiantado y la responsabilidad social de la universidad pública latinoamericana.

\section{VÍNCULOS ENTRE EL CONTEXTO UNIVERSITARIO Y EL QUEHACER DOCENTE}

Parte de los desafíos que enfrenta el contexto universitario actual y el quehacer docente, evidencia la necesidad de orientar la misión de la universidad hacia el servicio y diálogo social, la transferencia, la comunicación y la divulgación del conocimiento que permita contribuir en la solución de problemas complejos que afronta el ser humano (Gavrila, 2018; Predazzi, 2012). Aunque a nivel general la misión de la universidad pública ha sido fluida y dinámica en función de ideales filosóficos, políticos, culturales de las sociedades, actualmente existe la inminente necesidad de replantear las estrategias para atención a las necesidades individuales y sociales de las personas, pues en múltiples ocasiones se critica el hecho de que la misión de la universidad pública se redacta de manera superficial, al margen de la participación de la mayoría de los miembros de la organización, lo que conduce a un escaso impacto de la misión en el ámbito social, organizacional y en el quehacer de las personas docentes (Scott, 2006).

Por lo anterior, se considera que la universidad pública latinoamericana, como impulsora del bienestar social, debería apostar por el desarrollo humano de las personas, por ello es fundamental la acción de las unidades académicas para potenciar el aprendizaje significativo del estudiantado y el desarrollo ético del profesorado. En relación con lo anterior, el aprendizaje significativo se conceptualiza como "...una forma de aprendizaje consistente en activar experiencias y conocimientos previos con los que se relaciona e integra el nuevo conocimiento, en un proceso que implica atribución de significado o comprensión de conceptos" (Rivas, 2008, p. 28). Asimismo, para el desarrollo humano del profesorado universitario se requiere impulsar acciones para trasformar las prácticas de enseñanza, de forma que favorezcan el desarrollo del talento humano altamente efectivo y eficiente en el ejercicio de la docencia, la investigación y la acción social.

Desde lo expuesto en el párrafo anterior, el planteamiento de Paulo Freire constituye un discurso clásico que orienta de manera contundente la epistemología del quehacer docente, indistintamente del contexto o el nivel educativo donde se ejerza. Freire (2004), denota una postura ética y moral que la persona docente asume cuando ejerce; al interrelacionarse con el estudiantado en los diversos espacios y contextos educativos. En relación con lo anterior, el ejercicio de la docencia solo es posible, si existe una relación bidireccional de quien aprende y enseña. En este caso, para Freire (2004), tanto aprende quien enseña, como enseña quien aprende, por ello es necesario comprender que la enseñanza y el aprendizaje son indisolubles y dependen inexorablemente el uno del otro. Por lo antes expuesto el compromiso y la exigencia que el ejercicio de la docencia conllevaría como saber; dónde convergen la práctica y la teoría, que se nutren de una conciencia crítica y problematizadora de la persona docente y el estudiantado ante la realidad circundante. Aunque podría darse que no todas las personas docentes ejercen su rol desde este compromiso, 
ni tampoco resulta siempre exitoso el creer que la siempre las ventajas de una mejor docencia universitaria van a aminorar el fracaso del alumanado. Según Bourdieu y Passeron (2004), las problemáticas opresivas hacia el estudiantado no solamente están asociadas a limitaciones económicas o educativas sino también por el contexto sociocultural y las relaciones previas de estos con la cultura. Asimismo, Bourdieu (1997), cree que el estudiando menos aventajado culturalmente en relación con acceso a recursos económicos, bienes y servicios del Estado tiene ínfimas posibilidades de desarrollo en el contexto universitario, de ahí que contrario a lo que afirma Freire (2004), las condiciones materiales y económicas tienen mayor peso en el éxito estudiantil que el proceso educativo en sí mismo.

Como parte de estos desafíos, también surge la necesidad de formar al profesorado para que considere la planificación didáctica como la etapa fundamental para garantizar una buena enseñanza que se entiende como aquella acción educativa que coadyuva al logro de un aprendizaje significativo y a despertar la emoción del estudiantado, asimismo se pueden establecer mecanismos para que el ejercicio de la docencia tenga un papel preponderante dentro del quehacer de las facultades y se asignen a los mejores profesionales para desarrollar esta labor, con amplio conocimiento curricular, pedagógico y didáctico se constituye en uno de los desafíos más apremiantes (McMurtrie, 2019).

Todo lo anterior pone de manifiesto que, en el ejercicio de la docencia universitaria, se tiene como principal desafío el poder ofrecer desde la universidad una formación integral del ser humano que trascienda el conocimiento disciplinar en pro de una sociedad más justa y equitativa. Como lo menciona Freire (2004), el acto educativo trasciende el desarrollo de competencias disciplinares y se centra en una serie de habilidades necesarias para la vida de cualquier persona que cohabite con sus semejantes en un mundo con tantas desigualdades sociales.

Por otra parte, el diario ejercicio de la docencia universitaria debería tener un compromiso hacia potenciar el sentido crítico del estudiantado mediante reflexiones profundas sobre la vida misma y los elementos del contexto social y universitario. Por ello, se pueden generar acciones desde la práctica docente conducentes a elevar el nivel de curiosidad, de conciencia, de compromiso, de creatividad y de actitud hacia la vida para poder trascender de la ingenuidad a la crítica racional e irracional; sobre diversas problemáticas que a nivel personal y profesional atañen al ser humano. Sin embargo, que todo lo anterior implica un modelaje de acciones y actitudes por parte de la persona docente; ya que nadie es capaz de desarrollar capacidades que no posee o ejerce de manera natural.

Cabe considerar que las competencias, el pensamiento complejo y la interdisciplinariedad son condiciones que se establecen a partir de las características del contexto económico, político y social en general. Al mencionar elementos del contexto que propician la complejidad y el desarrollo de competencias para el trabajo en el ambiente universitario, se considera que en el mundo actual la globalización, la revolución tecnológica han provocado la masificación de información de baja calidad que no contribuye a la mejora sustancial de las problemáticas que se generan en el medio universitario (Scott, 2006).

En relación con lo anterior, la interdisciplinariedad entendida como la interacción entre diferentes disciplinas que permiten la generación de conocimiento desde diversas posturas epistemológicas, ontológicas y axiológicas favoreciendo la resolución de problemas complejos es un elemento que podría estar presente en la universidad pública latinoamericana para tener mayor impacto a lo interno de esta. Asimismo, en el ámbito de las actitudes para la vida y el trabajo, es necesario preparar a la población estudiantil para enfrentar los desafíos en los que se debatirá la humanidad a lo largo del siglo XXI. Por ello, se considera importante el planteamiento de diversos proyectos de investigación y programas educativos conformados por académicos de varias facultades. El enfoque interdisciplinario implica que se integren las diferentes disciplinas y métodos, lo que permite crear espacios para nuevas investigaciones, nuevas formas de docencia en educación superior (Culhane, Niewolny, Clark y Misyak, 2018), no obstante el trabajo interdisciplinario debe superar los escollos que se presentan en cuanto su credibilidad científica y aplicación práctica, ya que muchas veces las investigaciones o métodos que sustentan los proyectos carecen de suficiente anclaje epistemológico a una 
determinada disciplina lo que provoca que pierda credibilidad y valor ante la comunidad científica (Nissani, 1997).

En este sentido, cabe mencionar que la inteligencia artificial y la automatización de procesos generará millones de nuevos empleos para la fuerza laboral del mundo, rompiendo los paradigmas y esquemas hasta la fecha conocidos por el ser humano (Observatorio de Innovación Educativa, 2018; Cann, 2019). Según lo antes expuesto el problema que se deriva de la expansión de la inteligencia artificial es que requerirá de competencias cada vez más complejas para las personas formadas, que se tendrán que redefinir muchos puestos de trabajo y los perfiles de la fuerza laboral que hará frente a esa expansión de la tecnología y la ciencia de datos.

Las perspectivas que se derivan de la expansión de la inteligencia artificial requieren un nivel aceptable de competencias digitales por parte del profesorado universitario. La inteligencia artificial es un área de las Ciencias de la Computación que diseña sistemas inteligentes que emulan la capacidad cognoscente del cerebro humano para la resolución problemas y ejecución de tareas de alta complejidad (Ocaña, Valenzuela y Garro, 2019). En relación con lo anterior, los sistemas inteligentes bien podrían ser aprovechados para la mejora de la enseñanza y el aprendizaje aprovechando el potencial de la robótica y el internet de las cosas para generar innovación y valorar nuevas formas de aprender mediante las TD (Prendes, Gutiérrez y Martínez, 2018). En el ámbito antes descrito evidentemente el profesorado universitario tendrá que estar preparado para no caer en letargo cognitivo y actitudinal que impida el aprovechamiento del potencial que posee la tecnología digital, en este sentido Marcovitch (2002) afirma que "... la universidad jamás será una usuaria incondicional de las oportunidades creadas por la tecnología, pero desconocerla o dejar de aprovecharla, cuando se hace necesario, es absolutamente imperdonable" (p. 87). Por ello, los procesos de formación permanente en todo el mundo apuntan hacia modelos formativos que favorezcan no sólo la alfabetización digital del profesorado, sino también la reflexión en cuanto el uso pedagógico de la tecnología y a la didáctica específica del contenido disciplinar.

Otro de los grandes desafíos éticos de la universidad pública latinoamericana, consiste en impulsar la innovación educativa. Este reto es posible si las unidades de apoyo académico colaboran de forma significativa con el profesorado, creando espacios de trabajo colaborativo donde el profesorado tenga la posibilidad de experimentar y hacer innovación. Desde lo antes propuesto según Carvajal (2013), la innovación por medio del uso de la tecnología podría incentivarse en las facultades mediante: conferencias, trabajos multidisciplinarios entre académicos, exposiciones, talleres y creando comunidades de práctica y redes donde se puedan discutir aspectos vinculados con pedagogía, currículum, evaluación y gestión de la innovación, entre otros.

Respecto a las comunidades y redes académicas, en las últimas décadas la universidad como organización en constante expansión y desarrollo científico, ha promovido en conjunto con organizaciones gubernamentales y no gubernamentales diferentes propuestas para el desarrollo económico, político, social y planetario, mediante la creación de redes y comunidades académicas que desarrollan proyectos de diversa índole por medio de la colaboración entre las personas que integran las comunidades educativas, como por ejemplo la Red Docente de América Latina y el Caribe denominada Kipus o la Red Interuniversitaria Euroamericana de Investigación sobre Competencias Mediáticas para la Ciudadanía (Chapman, Pekol y Wilson, 2014; Francis y Cascante, 2011). Desde esta perspectiva, se maximizan los recursos económicos y se aúnan esfuerzos para la mejora a lo interno de las instituciones miembros, a la vez que se promueven iniciativas de orden global que inciden en diversos contextos universitarios.

La colaboración interuniversitaria, tiene diversas connotaciones y sirve para múltiples propósitos, de aquí que la concepción de red académica obedece a un conjunto de interconexiones, mecanismos de información y trasferencia de conocimiento que favorecen el trabajo colaborativo entre los grupos de personas con intereses comunes (Chapman et al, 2014). Estas redes se convierten en ecosistemas de aprendizaje que son fuertemente impulsados por las tecnologías digitales que permiten el envío y la recepción de información de 
manera organizada. En esta perspectiva, las redes académicas se convierten en sistemas complejos la gestión del conocimiento a nivel internacional y nacional, estimulado las posibilidades de desarrollo del capital humano, mejora del currículo universitario, internacionalización, innovación, cultura de trabajo, alianzas estrategias, resolución de problemas complejos, entre otros (Almuiñas y Galarza, 2016; Chapman, et al., 2014; Contreras, Ruiz, Vázquez y Salazar, 2013; Francis y Cascante, 2011).

Todo lo antes mencionado, promueve de alguna forma la colaboración participativa de diversas instituciones universitarias y agencias de cooperación internacional, gubernamentales y no gubernamentales. Dentro de los mayores beneficios que se obtienen por medio de las redes y comunidades académicas se encuentran los intercambios del estudiantado y del profesorado, así como la posibilidad de ejecutar programas y proyectos conjuntos entre universidades (Contreras, et al., 2013; Francis y Cascante, 2011). Asimismo, en cuanto a los beneficios para el desarrollo social, desde las redes y comunidades académicas se favorece el desarrollo social en proyectos que para los países u organizaciones son prioritarios, y en donde muchas veces no existen los recursos económicos para hacer frente al desarrollo e implementación de propuestas para la mejora o atención de problemas de diversa índole (Almuiñas y Galarza, 2016; Chapman, et al., 2014; Contreras, et al., 2013; Francis y Cascante, 2011). Todo lo expuesto en este apartado, sería posible si las unidades de apoyo académico colaboran de forma significativa con el profesorado, como por ejemplo creando espacios donde las personas docentes tengan la posibilidad de experimentar y hacer innovación. Desde lo antes propuesto la innovación podría incentivarse en las facultades mediante: conferencias, trabajo interdisciplinario entre académicos, exposiciones, talleres y creando comunidades de práctica donde se puedan discutir aspectos vinculados con currículo, pedagogía, didáctica y evaluación, entre otros.

\section{VALORES ÉTICOS Y DESARROLLO DEL CURRÍCULO UNIVERSITARIO}

En la actualidad, para la comunidad universitaria es necesario investigar sobre los diferentes cambios en el ámbito social, con el fin de orientar las propuestas curriculares hacia una trasformación social, con el protagonismo de la universidad pública latinoamericana como institución formadora de ciudadanos con sólidos conocimientos disciplinares, pero con un sentido humano y social. Por ello, la universidad pública latinoamericana tendrá que ser cercana a los enfoques curriculares de corte humanista que impliquen compromisos con el desarrollo integral de las personas, que vayan más allá de los contenidos conceptuales y procedimentales. Todo esto, enmarca al currículo como un concepto que evoluciona y se permea de diferentes elementos culturales, corrientes filosóficas, teorías del aprendizaje que de una u otra forma ha orientado el quehacer curricular. Estos planteamientos dan origen a diversos enfoques curriculares que orientan el desarrollo de programas formativos dentro de la universidad.

En relación con lo anterior, dada la diversidad de disciplinas que convergen en el ámbito universitario, las tendencias del currículo se establecen a partir de las necesidades del contexto disciplinar y las diferentes políticas universitarias en cuanto a la conformación de los programas de estudio y las condiciones específicas, como la internacionalización universitaria. Asimismo, el currículo se concibe como un proyecto institucional donde convergen aspectos formativos, ideológicos y políticos (Giraldo, Flórez y Cadavid, 2012). Por ello, en el proyecto curricular universitario tiene un gran peso la conformación de trayectos de formación integral que favorezcan el desarrollo pleno de las personas.

Conforme a los diferentes enfoques curriculares en la universidad, el concepto de currículo se presenta como proyecto formativo integral de las personas que se asocia con el desarrollo histórico y social que evoluciona de acuerdo con los avances sociales, científicos y las necesidades humanas (Addine citada por Vila, 2011). Desde lo antes planteado, es importante anotar que las personas actoras implicadas en el proceso formativo colaboran de manera proactiva hacia la conformación de consensos que permitan normar y organizar el currículo institucional. Asimismo, el currículo se puede presentar de manera oficial u oculta en las aulas universitarias (Posner, 2005), por esto muchas veces el currículo oficial se diluye o distorsiona con 
la acción educativa ejercida por la persona docente, provocando que el estudiantado no cuente con el perfil de salida declarado en los programas de estudio.

Por otro lado, existen diversas posiciones respecto a la relación entre el currículo, la pedagogía y la didáctica, por ello es difícil delinear los límites entre los tres conceptos, no obstante la pedagogía "...tiene como objetivo el diseño de experiencias culturales que conduzcan [...] al progreso de las personas y en el desarrollo de sus potencialidades [...] con miras a su formación" (Flórez, 2000, p. XIX) y la didáctica, está vinculada con "...las metodologías de enseñanza, al conjunto de métodos y técnicas que permiten enseñar con eficacia" (Flórez, 2000, p. XXI).

Por otra parte, en cuanto a los diferentes enfoques curriculares constituyen un conjunto de principios teóricos y prácticos que orientan el quehacer educativo en la universidad (Giraldo et al., 2012). Asimismo, estos se nutren de las diferentes teorías del aprendizaje, las fuentes filosóficas y las corrientes de producción del mercado. Ahora bien, el currículo universitario plantea una serie de componentes que orientan el proceso formativo de principio a fin. Los componentes antes mencionados están vinculados con la concepción de los objetivos de aprendizaje o metas que se pretenden alcanzar, los aspectos epistemológicos que orientan el proceso de enseñanza y aprendizaje, que responde a preguntas como: ¿Qué se enseña?, ¿qué se aprende?, ¿cómo se enseña?, ¿para qué se aprende?, entre otros cuestionamientos.

Cabe considerar que, dentro de los enfoques curriculares, los elementos más comunes están relacionados con las características de los procesos de enseñanza y aprendizaje, los recursos didácticos, la evaluación, la estructura de los programas y cursos, la modalidad educativa mediante la cual se ofrecerá el programa, entre otros aspectos. En el mismo orden de las ideas anteriores, en cuanto a la conformación de los planes de estudios y su relación con los programas del curso, el currículo como proyecto formativo, requiere de la conformación de planes de estudio que concreten el sentido de formación que la universidad quiere ofrecer al estudiantado. Por ello, en este proceso surgen una serie de interrogantes vinculadas con el tipo se ser humano que se quiere formar y cuáles son las condiciones básicas de la disciplina a la que está ligada dicha formación (Flórez, 2000; Giraldo et al., 2012).

Desde lo antes descrito, se puede decir que estas aspiraciones promueven trayectos formativos diversos, atendiendo la internacionalización de la universidad, las demandas del mercado, las políticas del Estado, entre otros aspectos que se pueden considerar. En el planteamiento de los programas de estudio, también intervienen las diferentes formas en que se desarrolla el currículo y las normativas en cuanto al modelo de educación que ofrece la universidad, las modalidades educativas disponibles, la distribución de los periodos académicos y horarios, la normativa académica, entre otros aspectos.

Una vez analizados y definidos los aspectos mencionados anteriormente, para la creación de un programa es necesario definir el perfil de las personas a las cuales se dirige la formación, esta definición no suele ser una tarea trivial porque implica cuestionarse qué condiciones específicas deben tener las personas que ingresan al plan de formación (perfil de entrada) y bajo cuáles condiciones se dará la salida profesional, una vez que las personas superen el trayecto formativo (perfil de salida). Estas condiciones serán identificadas y analizadas a partir de las prioridades y tendencias en cuanto a la formación disciplinar y los consensos que se logren alcanzar entre los diferentes actores involucrados (Hawes, 2010; Zabalza, 2010).

Otro elemento vinculado con los planes de estudios y programas del curso es la definición de contenidos, los cuales son generales, disciplinares de especialización y prácticas profesionales. Dichos contenidos, tienen una incidencia directa sobre el marco organizativo de la malla curricular y la organización de los cursos, generalmente la universidad tiene definidos la cantidad de créditos para cursos de diversa naturaleza, la distribución horaria y los ciclos académicos. Por último, para las unidades académicas es importante considerar el nivel de compromiso y participación que asume el profesorado en la elaboración de los programas de curso, de tal manera que se evidencie un trayecto formativo coherente y alineado con la propuesta curricular. 


\section{DESARROLLO DE LA FORMACIÓN INTEGRAL DEL ESTUDIANTADO}

La formación integral del estudiantado es un concepto amplio y multidimensional que implica una reflexión profunda sobre el ser humano y su entorno. Desde la perspectiva aquí adoptada, la formación integral se define como un proceso que permite la reflexión profunda sobre el propio ser y el contexto circundante para el bienestar propio, el de las personas semejantes y de los otros seres vivos que cohabitan en el entorno (Gadamer, 2000).). En otras palabras, las personas tienen la capacidad de construir la propia existencia a partir de todo lo que les rodea (Gadamer, 2000; García, 2007). En todo lo anterior, interviene la autodeterminación y la actitud con la que se asuma la responsabilidad formativa, por ello, parte del compromiso de la formación recae sobre la persona estudiante, pero también sobre el profesorado y la universidad como institución que facilita la formación integral.

Como se ha mencionado, la universidad pública no permanece ajena a los fenómenos propios de la sociedad postmoderna que la impulsa a sucumbir a la idea de ser una empresa más (Pizzul, 2013). Por lo tanto, en el ámbito social, como al interior de las mismas universidades hay elementos disruptivos que se contraponen a una formación integral y humanista. En este sentido, la globalización planetaria y el modelo económico capitalista impulsado por los organismos internacionales promueven la industrialización y la masificación de bienes de consumo. Asimismo, existe una tendencia marcada a nivel de políticas públicas que, poco a poco, ha reducido los recursos económicos con los que cuenta la universidad pública latinoamericana para hacerle frente al gran reto de proveer de una formación integral al estudiantado y atender las necesidades sociales. Por ello, se cierne sobre la universidad la amenaza latente de abandonar su misión histórica para asumir el rol de empresas, reduciendo su acción a la producción de bienes y prestación de servicios con fines de lucro.

Ante el panorama antes descrito, la persona docente como modelador en el proceso formativo integral tiene un papel fundamental en la conformación de la realidad social. En este sentido, la persona docente orienta el proceso formativo y lo enriquece con los contenidos conceptuales y procedimentales, pero con gran énfasis en las actitudes que conforman las emociones del ser humano. El pleno conocimiento de la verdad como constructo social involucra el razonamiento, la argumentación y la reflexión que trasciende el currículo universitario y sitúa al estudiantado en la vida misma y las responsabilidades éticas, morales y espirituales asumidas primigeniamente en su propio ser, con las personas semejantes y con la sociedad en general. Asimismo, en la búsqueda de la verdad, el alumnado debe tener la disposición de asumir una actitud abierta y propositiva hacia el aprendizaje y la reflexión, para develar la realidad circundante y la de su propio ser (Pizzul, 2013).

La formación más allá de un campo disciplinar, plantea el reto de visualizar el acto formativo como un proceso integral que forjará el carácter y las habilidades de convivencia de las personas a lo largo del ciclo de vida. Ahora bien, en los trayectos formativos del estudiantado en la universidad, se da más énfasis a la formación profesional, y se diluye o desdibuja la formación orientada al desarrollo actitudinal y a los aspectos de la cultura que determinan al ser humano como persona. En este sentido, la exclusión del desarrollo actitudinal y de los valores de la cultura en la formación profesional del estudiantado limita el desarrollo cognitivo, la toma de decisiones y la capacidad de resolver conflictos tanto a nivel personal como profesional (Vázquez y Manassero, 2007). Por ello, en la búsqueda del perfeccionamiento y la formación rigurosa en el ámbito disciplinar, muchas veces está ausente el producir o investigar sobre aspectos que implican reflexionar sobre los compromisos éticos y sociales que se asumen cuando se ejerce una determinada profesión.

El trayecto formativo que permite el desarrollo integral de las personas facilita el acercamiento al ser, que favorece una interacción que coadyuva a la reflexión profunda sobre los acontecimientos personales, familiares, comunitarios, sociales, económicos, políticos e ideológicos, entre otros que inciden en el comportamiento humano y que determinan la construcción de la personalidad y el desempeño profesional en el futuro. Aunado a lo anterior, es fundamental promover los espacios dentro y fuera de las aulas universitarias 
para que se puedan obtener de las experiencias cotidianas aprendizajes y conocimientos que faculten el ejercicio profesional y el desarrollo humano de la persona, las familias y la sociedad en general (Pizzul, 2013).

Aunque cada vez existen más elementos disruptivos como la disminución del presupuesto, los altos índices de interinazgo y la precarización de los salarios del profesorado, que se contraponen a la docencia, la investigación y la acción social como misión histórica de la universidad, según Pizzul (2013), es necesario que los valores humanos no sean olvidados por las personas actoras que intervienen en el proceso educativo. Entender el compromiso de preservar los valores humanos, también implica reflexionar sobre el papel que tiene la universidad pública latinoamericana frente a las constantes amenazas por parte del modelo económico capitalista y las debilidades internas como por ejemplo estabilidad docente, infraestructura adecuada, sistemas de información acordes con las necesidades de la comunidad universitaria, recursos necesarios para la investigación y la extensión, que impulsan a las instituciones de educación superior a convertirse en instrumentos para satisfacer las demandas mercantilistas.

La formación integral permite generar conciencia humana sobre las acciones en el ejercicio de una determinada profesión y en el entorno en que se vive. Dicha formación no riñe con la rigurosidad en la formación profesional, sino más bien complementa el desarrollo humano a partir de la reflexión sistemática sobre el sentido de la vida y el bienestar en el ejercicio profesional y en la vida cotidiana. Con lo antes descrito, se resignifica el concepto de formación profesional, más allá de los contenidos conceptuales y procedimentales de una disciplina, y se centra en develar todas aquellas acciones humanas que permiten el desarrollo pleno e integral del saber ser. En este sentido, la formación integral desvincule a las personas de los estándares y patrones creados por la sociedad de consumo para la enajenación del ser humano, es por lo que la formación integral surge como una esperanza para hacer frente a los problemas que platea la sociedad actual (D' Antoni, 2013; Freire, 2004; Gadamer, 2000; García, 2007; Pizzul, 2013).

Por otro lado, desde la mediación y la interacción persona docente-persona estudiante, es necesario enriquecer el acto educativo con actividades de mediación intencionadas para generar una reflexión profunda sobre las emociones y eventos que acontecen alrededor de las personas. Este tipo de actividades exigen una planificación con temáticas y actividades que despierten el interés y la curiosidad por parte del estudiantado. Asimismo, el modelaje que el profesorado desarrolle en su accionar, es determinante para generar conciencia crítica y reflexiva en la formación integral de las personas. Todo lo anterior, implicará una introspección de la persona docente hacia su propio ser, tanto en el plano personal como profesional. En este caso, el aprendizaje del estudiantado se favorece a partir de la observación de la actuación del profesorado en situaciones reales que repercuten significativamente en el la adquisición de valores y prácticas de comportamiento para el bienestar colectivo (Bandura, 1987). Para el profesorado y el estudiantado son necesarios los espacios académicos dentro de la universidad para discutir sobre las implicaciones éticas y morales que subyacen al ejercicio de una profesión. Asimismo, es importante para la formación integral del estudiantado resaltar el papel que tienen las humanidades para la conformación de la conciencia personal y social.

Por otro lado, desde los proyectos de extensión y de investigación se debe favorecer el desarrollo de valores y acciones de alto valor social que permitan evidenciar la importancia que tiene para el ser humano el desarrollo de habilidades de convivencia y de comunicación asertiva. Lo anterior, refuerza el papel histórico de la extensión e investigación en la universidad pública latinoamericana, como medio de cambio para promover el desarrollo de las libertades y valores en comunidades generalmente vulnerables (Zlateva, 2017), posicionándose como una instancia determinante para el desarrollo humano en las comunidades beneficiadas.

\section{COMPROMISOS Y RESPONSABILIDAD SOCIAL DE LA UNIVERSIDAD PÚBLICA LATINOAMERICANA}

La responsabilidad social, entendida como aquellos compromisos promovidos desde el quehacer universitario para mejorar los condiciones de las personas dentro y fuera de esta, mediante la promoción de la 
trasparencia y la ética canalizada en proyectos educativos para el desarrollo del bienestar individual y colectivo tomado en cuenta las expectativas y necesidades comunitarias (Vallaeys, 2016). Lo anterior, se evidencia por medio la participación en el desarrollo comunal y atención a las problemáticas sociales abordadas desde la colaboración y compromiso del estudiantado y profesorado, con el apoyo incondicional de las personas que habitan en las comunidades a las que se dirigen los proyectos.

Desde la planificación institucional de la universidad pública latinoamericana es determinante afianzar el desarrollo de proyectos que generen impacto social y una conciencia ciudadana del estudiantado participante. En este sentido, la universidad pública latinoamericana, mediante la acción social, proyecta en áreas seleccionadas, partiendo de un proceso de diálogo social y diagnósticos que permita identificar problemáticas que afectan significativamente a la sociedad. Asimismo, para Vallaeys (2016) la acción social y el compromiso ético asumido por la universidad pública latinoamericana, va más allá de activismos que conduzcan al cumplimiento de los requisitos para obtener una titulación universitaria por parte del estudiantado.

La democratización y el servicio social fueron promovidos como una gran aspiración de la educación superior a partir del siglo XVII, principalmente por las universidades estadounidenses, quienes impulsaron la premisa de que los ideales democráticos implican la atención a las necesidades individuales y sociales de las personas (Scott, 2006). Asimismo, en América Latina mediante la Reforma Universitaria de Córdoba, Argentina plateada en 1918, se sentaron los pilares para redefinir la acción de la universidad pública latinoamericana en función de los principios democráticos, el compromiso social y los derechos humanos (Del Huerto, 2018). En este sentido, la responsabilidad social de la universidad pública se enfoca tanto al cuidado de la propia comunidad universitaria como también de la comunidad nacional y para ello se requiere de una adecuada gestión, investigación, calidad, ética y democratización del proceso.

Considerando lo expuesto en el párrafo anterior, la responsabilidad social es considerada como una iniciativa que atiende tanto los problemas externos a la universidad como también los internos, ya que la reciprocidad en el aprendizaje es mutua, por lo tanto, el beneficios de las acciones sociales repercute en la mejora de las problemáticas internas y externas de la universidad (Vallaeys, 2016). Esta tarea implica un alto nivel de compromiso e investigación, ya que, en muchas ocasiones no se tienen respuestas certeras a todas las situaciones disruptivas que inciden negativamente en la sociedad y la universidad.

La responsabilidad social de la universidad pública latinoamericana se promueve mediante mejoras organizacionales, educativas, cognitivas y sociales. Desde lo antes descrito, al abordar el tema organizacional, la universidad como institución pública puede procurar el mejoramiento de las condiciones medioambientales, laborales del personal y de bienestar del estudiantado en general. En todo momento, la universidad deberá ser un referente ético y democrático, consecuente con la misión y la visión (Vallaeys, 2016).

Por otro lado, la responsabilidad social implica un alto compromiso con la formación integral del estudiantado. Dicha formación puede estar reflejada en el accionar del estudiantado frente a las condiciones sociales. Por otro lado, el trayecto formativo impulsado desde la universidad debe proveer al estudiantado las capacidades para efectuar la autogestión del conocimiento con miras al ejercicio profesional, pero también para una participación social solidaria, eficiente y activa. En relación con lo anterior, el sentido de la acción social universitaria se gestionaría garantizando la comunicación oportuna de los proyectos sociales más apremiantes y su impacto en el ámbito social e institucional.

Para articular el proceso que conduce a la formulación, desarrollo e implementación de los proyectos de acción social, partiendo de un proceso diagnóstico que favorezca el establecimiento de las necesidades y aspectos de contexto vinculados con la problemática social o institucional que se quiere abordar. Con respecto a lo anterior, para las unidades académicas es importante planificar la evaluación de los principales logros de la acción social en cuanto a las metas establecidas inicialmente y a detallar aspectos de mejora debido a la necesidad de evidenciar la eficiencia en el uso de los recursos económicos y la efectividad de las acciones emprendidas. Cabe destacar, que las acciones en torno a la acción social deben ser planificadas a largo plazo, 
de manera que se pueda dar una efectiva continuidad a los proyectos que se emprenden desde las diferentes unidades académicas (Vallaeys, 2016).

En relación con lo anterior, las actividades, evaluaciones y logros pueden ser socializados con la comunidad universitaria y las personas actoras sociales participantes. En este caso, las redes sociales representan una herramienta para garantizar el acceso y la divulgación de los logros más importante que se obtienen a partir de los proyectos sociales. De igual manera, se considera que mediante de las tecnologías digitales se puede favorecer la interacción y el trabajo colaborativo que puede prevalecer entre las personas participantes cuando se organizan proyectos de responsabilidad social universitaria.

Por otra parte, las acciones destinadas a la mejora significativa de las problemáticas sociales serán sostenibles y permanentes en el tiempo, mientras se mantengan las condiciones adversas que les dieron origen, de esta forma, desde la sociedad, se dará un sentido a la misión de la universidad pública y al quehacer de la educación superior, si los resultados de las acciones derivadas de la enseñanza, la investigación y la acción social promueven trasformaciones positivas que se evidencian en productos tangibles que coadyuvan a la mejora de problemáticas complejas que la sociedad enfrenta. Por último, que solamente con una fuerte sinergia entre la universidad y otros organizaciones sociales será posible garantizar el ejercicio democrático de los derechos humanos y la mejora sustancial, tanto del entorno universitario como del contexto social.

\section{CONSIDERACIONES FINALES}

Según lo planteado en los apartados anteriores, la universidad pública latinoamericana como institución social puede renovarse y adaptarse a las transformaciones que están ocurriendo en el mundo para poder garantizar una mejor educación y proyección social. Todo lo anterior, propicia la búsqueda de afinidades entre los intereses de diversos grupos sociales y la educación superior, pues para la universidad el acercamiento a grupos sociales fuera de esta implica una aproximación al mundo y las prácticas de la vida real de los seres humanos. Por otro lado, desde fuera de la universidad se podría encontrar sentido a la misión de esta y al quehacer de la educación superior pública latinoamericana, si los resultados de la enseñanza, la investigación, la acción social, la nacionalización, la democratización y la internacionalización promueven trasformaciones positivas que se evidencian en productos tangibles que coadyuven a la mejora de problemáticas complejas que la sociedad enfrenta.

En relación con lo anterior, desde los ámbitos universitarios se aprende a enseñar para incluir, enseñar para vivir con las personas semejantes y enseñar a los seres humanos con diferentes condiciones de educabilidad. Por ello, es necesario el desarrollo de competencias cognitivas y humanas donde las personas sean capaces de pensar, analizar y resolver problemas; debe existir una apropiación de la investigación, del currículo, la pedagogía y la didáctica que produzca transformaciones para organizar la acción universitaria ante las demandas sociales.

Desde la ética en desarrollo del currículo universitario contemple aspectos relacionados con temas de interés social, político y tecnológico para satisfacer las necesidades que las personas deben enfrentar en el entorno actual. En relación con lo anterior, la enseñanza podría incorporar nuevas herramientas y medios que atiendan a las nuevas competencias que exige el contexto, pero al mismo tiempo haciendo hincapié en los individualismos de cada persona, por ello desde la universidad se deberán atender los nuevos principios o bases curriculares que requiere la sociedad actual y velar porque estos se desarrollen eficazmente. Todo lo anterior, requiere de un trabajo articulado, teniendo en cuenta las necesidades específicas de la comunidad universitaria y los mecanismos para el fomento del trabajo colaborativo e interdisciplinario con los diversos grupos sociales. En este sentido, cabe resaltar que los currículos tradicionales y los conocimientos adquiridos por las personas docentes son limitados en algunos casos; por tanto, la educación superior se enfrenta a grandes desafíos como el contar con mecanismos que permitan la formación permanente del personal docente, la investigación y el contacto directo con las necesidades de la sociedad. 
La ética en el ámbito curricular implica para el profesorado la búsqueda de recursos didácticos del aprendizaje que despierten el interés por la argumentación y la reflexión sistemática del estudiantado hacia los problemas sociales y del área disciplinar. Asimismo, se podrían concretar acciones que permitan apoyar al alumnado en el trayecto formativo, de tal forma que siempre cuenten con los apoyos pedagógicos y didácticos para lograr con éxito el perfil de salida que se pretende lograr.

En relación con lo descrito en el párrafo anterior, no menos relevante es el considerar la evaluación permanente de los programas de estudio y de los cursos, esto permitirá efectuar ajustes y enriquecer los procesos de reflexión en relación con los resultados que se pretenden obtener durante la formación del estudiantado. Asimismo, la evaluación de los procesos implicados en el diseño curricular facilitará el perfeccionamiento de estos. En este caso, se buscan alternativas flexibles que permitan la actualización permanente de los programas de estudio y sílabos de los cursos. En todo lo anterior interviene la actitud con la que se asuma dicha responsabilidad por parte del equipo gestor de las propuestas curriculares.

Es posible afianzar las propuestas curriculares universitarias con contenidos actitudinales, donde se evidencie una formación humanista, que favorezca el desarrollo de la persona y del entorno social más allá de los conocimientos y habilidades de la disciplina. Por otro lado, las unidades académicas responsables de los planes de formación deberán procurar la creación de apoyos y actividades de formación complementaria que requiere el estudiando para lograr el perfil de salida propuesto. Respecto a lo anterior, una de las principales críticas que hace Pino (2005), en relación con la reflexión planteada en el párrafo precedente, es que justamente en los currículos de los programas de formación universitarios se evidencia una visión reduccionista e instrumental de la acción educativa, lo que provoca que se dejen de lado las construcciones culturales que conectan el currículo con los problemas sociales.

Aunado a lo dicho, el currículo universitario conduce a la formación de las personas con capacidades que coadyuven con la autogestión del conocimiento. Desde lo antes planteado, también el desarrollo humano del profesorado es estratégico para trasformar las prácticas de enseñanza, que permita el ejercicio pleno de la docencia universitaria, la investigación y la acción social. Por esto, la formación permanente del profesorado constituye en uno de los desafíos más apremiantes para las escuelas y unidades académicas dentro de la universidad.

Respecto a la práctica cotidiana del quehacer del profesorado universitario, interesa enfocar la postura de Freire (2004), respecto a la critica. Esta exigencia, permite trascender de la ingenuidad sobre una realidad concreta a la puesta en marcha de cuestionamientos que permitan generar un conocimiento más profundo de la realidad respecto a las condiciones políticas, económicas, socioculturales y ambientales que inciden sobre la calidad de vida del ser humano, y en este sentido la educación debería potenciar la curiosidad indagadora que estimule la generación del sentido crítico y la creatividad de las personas.

El gran reto que afronta el profesorado universitario respecto a lo anterior radica en el establecimiento de acciones concretas de mediación que develen realidades sociales y académicas para estimular los cuestionamientos, la reflexión y el auto análisis en relación con los aspectos satisfechos e insatisfechos de la vida, entre otros factores que se podrían abordar desde el enseñar y el aprender. Por ello, se pone principal atención y mirada crítica ante propuestas sociales y científicas que intentan MacDonalizar la sociedad, mediante el establecimiento de verdades absolutas (Ritzer, 1996), esta es una de las tareas más apremiantes del sentido crítico, ético y moral que toda persona docente debe asumir como exigencia ante la realidad.

El estudiantado que asiste a la universidad pública en América Latina ve en la educación superior una posibilidad de salir de los índices de pobreza y brechas que provoca el sistema económico neoliberal. No obstante, muchas de las personas que se encuentran bajo los índices de pobreza nunca logran ingresar a la universidad. Aunque la universidad pública al igual que otras instituciones sociales reproducen patrones de opresión, dominación y desigualdad; paradójicamente es desde estas mismas instancias donde se impulsan las acciones para combatir la pobreza y la desigualdad (D' Antoni, 2013). No queda otro remedio que luchar hasta el final por la justicia social que tanto se anhela desde la universidad (D' Antoni, 2013). Lo anterior, 
solo es posible con la acción de las personas docentes y el impulso de políticas educativas que defiendan el sentido de la educación como un agente de trasformación social y desarrollo humano.

La acción social universitaria puede responder a la misión y la visión de la universidad pública latinoamericana directamente relacionada con la "...formación integral profesional y ciudadana y compromiso social con el desarrollo" (Vallaeys, 2016, p. 158). En este sentido, las condiciones que garantizan una responsabilidad social universitaria que tenga impacto, van más allá del simple activismo y los requisitos académicos; están vinculadas con los valores éticos y democráticos en el accionar universitario.

Aunque los grupos de poder intenten justificar sus acciones desde el discurso de dominación social que hace creer que el mundo es justo o atribuyendo la desigualdad y la miseria a la autoestima de las personas, es inminente tomar acciones, en primera instancia desde lo personal y lo familiar, pero posteriormente desde lo colectivo (García, 2010). Esto implicará, un mayor compromiso por parte de la universidad pública latinoamericana para promover de manera real y efectiva acciones que coadyuven al mejoramiento de las condiciones económicas y afectivas de las personas con mayores necesidades. Todo lo anterior implicará la redefinición de la distribución del presupuesto universitario, así como mecanismos para favorecer la acción social desde los diversos ámbitos educativos. Por ello, es esperanzador para América Latina que, aunque las condiciones económicas de la región son deplorables y entre los países existen desigualdades y diferencias irreconciliables, en los últimos años se han impulsado políticas para el desarrollo humano que de una u otra manera han beneficiado al estudiantado con una educación pública universitaria gratuita o de bajo costo como es el caso de Argentina, Cuba, Uruguay, Costa Rica y Chile, entre otros (Comboni y Juárez, 1997).

Para finalizar, en el futuro se debe conjeturar sobre los desafíos del currículo universitario considerando aspectos relacionados con temas de interés social, político, tecnológico, entre otros para satisfacer las necesidades que las personas deben de enfrentar en el entorno actual. En relación con lo anterior, es pertinente discutir si la enseñanza debe incorporar nuevas herramientas y medios que atiendan a las competencias laborales que exige el contexto global, y al mismo tiempo hacer hincapié en los individualismos de cada persona, por ello desde la universidad pública latinoamericana deberán plantarse nuevos principios o bases curriculares que requiere la sociedad actual y velar porque estos se desarrollen eficazmente. Todo lo anterior sin duda, requiere de un trabajo articulado teniendo en cuenta las necesidades específicas de la comunidad universitaria y los mecanismos para el fomento del trabajo colaborativo e interdisciplinario con los diversos grupo sociales.

\section{REFERENCIAS}

Almuiñas, J. y Galarza, J. (2016). Las redes académicas como ejes de integración y cooperación internacional de las instituciones de educación superior. Revista Cubana de Educación Superior, 35(1), 18-29. Recuperado de http s://bit.ly/38ghkI 4

Bandura, A. (1987). Pensamiento y Acción, fundamentos sociales. (2. ed.). Barcelona: Ediciones Martínez Roca.

Bourdieu, P. (1997). Capital cultural, escuela y espacio social. México, D. F: Siglo XXI.

Bourdieu, P., y Passeron, J. C. (2004). Los Herederos: los estudiantes y la cultura. Buenos Aires: Siglo XXI.

Bunge, M. (2005). Diccionario de filosofía. (3ª ed. en español) México D.F: Siglo XXI

Cann, O. (2019). Report on the Future of the Works. Ginebra: World Economic Forum. Recuperado de https://bit. ly/32E0nX2

Carvajal, V. (2013). Modelo pedagógico para el desarrollo de programas educativos con componente virtual, dirigidos a adultos de zonas rurales centroamericanas (Tesis de Doctorado).Universitat de les Illes Balears, Illes Balears, España. Recuperado de https://bit.ly/32HtEQL

Chapman, D., Pekol, A., y Wilson, E. (2014). Cross-border university networks as a development strategy: Lessons from three university networks focused on emerging pandemic threats. International Review of Education, $60(5)$, 619-637. Recuperado de https://bit.ly/3cAfAgq 
Comboni, S., y Juárez, J. M. (1997). La educación superior en América Latina: perspectivas frente al siglo XXI. Política y Cultura, (6), 7-27. Recuperado de https://bit.ly/2IhyvhO

Contreras, S., Ruiz J., Vázquez, E., y Salazar, F. (2013). Redes académicas de investigación. Apertura, 4(2), 144-155. Recuperado de https://bit.ly/2I7NLxJ

Culhane, J., Niewolny, K., Clark, S., y Misyak, S. (2018). Exploring the Intersections of Interdisciplinary Teaching, Experiential Learning, and Community Engagement: A Case Study of Service Learning in Practice. International Journal of Teaching \& Learning in Higher Education, 30(3), 412-422. Recuperado de https://bit.ly/2vBeFLM

D’ Antoni, M. (2013). Educación superior en Costa Rica: ¿"Rankización” y descolonización?. Revista Electrónica Educare, 17(3), 21-40. Recuperado de https://bit.ly/39dX3Eq

Del Huerto, M. (2018). La Reforma universitaria de Córdoba: pionera en el legado latinoamericano de universidad popular. Revista Cubana de Educación Superior, 37(1), 37-47. Recuperado de https://bit.ly/2TieJt0

Flórez, R. (2000). Evaluación pedagógica y cognición. Colombia: McGraw Hill.

Francis, S., y Cascante, N. (2011). Aproximaciones teórico-conceptuales para comprender la relación de comunidades académicas con el saber pedagógico del docente universitario. Actualidades Investigativas en Educación, 10(1), 1-13. Recuperado de https://bit.ly/2VCkDqm

Freire, P. (2004). Pedagogía de la Autonomía: Saberes Necesarios para la Práctica Educativa. Sao Pablo, Brasil: Paz e Terra S. A.

Gadamer, H. G. (2000). La educación es educarse. Barcelona: Paidós.

García, J. (2010). Ideología de la Desigualdad: Análisis de la Investigación Empírica en Psicología Social. Revista Electrónica de Psicología Política, 8(24), 67-87. Recuperado de https://bit.ly/2Tdlzjp

García, M. (2007). Las nociones de formación en los investigadores del Instituto Superior de Ciencias de la Educación del Estado de México. (Tesis de Doctorado). Universidad Pedagógica Nacional, México. Recuperado de https://bi t.ly/2PGAu3y

Gavrila, M. (2018). A new direction in university. Teaching between solidarity, complexity and media education. Italian Journal of Sociology of Education, 10(2), 57-75. Recuperado de https://bit.ly/2If8wry

Giraldo, E., Flórez, S., y Cadavid, A. (2012). Enfoques curriculares: Orientaciones y perspectivas en las propuestas de formación de maestros/as. Revista Educación y Pedagogia, 24(63), 74-90. Recuperado de https://bit.ly/38cOJUi

Hawes, G. (2010). Del perfil de egreso a la malla curricular: Propuesta general metodológica para el diseño de un plan de formación profesional universitaria. Santiago: Universidad de Chile.

Marcovitch, J. (2002). La universidad (im)posible. Cambridge: Cambridge University Press.

McMurtrie, B. (21 de marzo de 2019). Many Professors Want to Change Their Teaching but Don't. One University Found Out Why. The Chronicle of Higher Education. Recuperado de https://bit.ly/38d3ZjX

Nissani, M. (1997). Ten Cheers for Interdisciplinarity: The Case for Interdisciplinary Knowledge and Research. Social Science Journal, 34(2), 201-216. Recuperado de https://bit.ly/2Trr1y4

Observatorio de Innovación Educativa. (2018). Los retos de la fuerza laboral frente a la automatización. Nuevo León: Tecnológico de Monterrey. Recuperado de https://bit.ly/2TrYAAg

Ocaña, Y., Valenzuela, L., y Garro, L. (2019). Inteligencia artificial y sus implicaciones en la educación superior. Propósitos y Representaciones, 7(2), 536-568. Recuperado de https://bit.ly/3akVrZS

Pino., S. (2005). El Desarrollo Humano como eje transversal de las estructuras curriculares. Revista Electrónica de la Red de Investigación Educativa, 1(2), 1-12. Recuperado de https://bit.ly/2TwfYUi

Pizzul, M. (2013). La formación humanistica, fin esencial de la Universidad. Mar del Plata, Argentina: Universidad FASTA. Recuperado de https://bit.ly/2vlBHqb

Posner, G. (2005). Análisis de Currículo (3a ed.). México, D.F.: McGraw-Hill.

Predazzi, E. (2012). The third mission of the university. Rendiconti Lincei, 23(1), 17-22. Recuperado de https://bit .ly/2Tgz95Q

Prendes, M., Gutiérrez, I., y Martínez, F. (2018).Competencia digital: una necesidad del profesorado universitario en el siglo XXI. Revista de Educación a Distancia, (56), 1-22. Recuperado de https://bit.ly/3crVuVy 
Manuel Baltodano Enríquez. Perspectivas éticas de la docencia universitaria en América Latina: ret...

Ritzer, G. (1996). La McDonalización de la sociedad: Un análisis de la racionalización en la vida cotidiana. Barcelona: Ariel Sociedad Económica.

Rivas, M. (2008). Procesos cognitivos y aprendizaje significativo. Madrid: Consejería de Educación.

Sánchez, A. (1974). Ética. (10a ed.). México: Grijalbo.

Scott, J. (2006). The Mission of the University: Medieval to Postmodern Transformations. Journal of Higher Education, 77(1), 1-39. Recuperado de https://bit.ly/3ao5ihu

Vallaeys, F. (2016). Introducción a la Responsabilidad Social Universitaria RSU. Barranquilla: Ediciones Universidad Simón Bolívar. Recuperado de https://bit.ly/39ikrk4

Vázquez, A., y Manassero, M. (2007). En defensa de las actitudes y emociones en la educación científica (I): evidencias y argumentos generales. Revista Eureka sobre Enseñanza y Divulgación de las Ciencias, 4(2), 247-271. Recuperado de https://bit.ly/2TyvoY6

Vila, D. (2011). Las concepciones de curriculum y su importancia en la elaboración de un doctorado curricular en estomatología. Revista Cubana de Estomatología, 48(3), 301-314. Recuperado de https://bit.ly/2VwYqKu

Zabalza, M. (2010). Competencias docentes del profesorado universitario: Calidad y desarrollo profesional. Madrid: Narcea Ediciones.

Zlateva, P. (2017). La contribución de la extensión universitaria al desarrollo humano y a la expansión de las capacidades. Análisis de proyectos de la Universidad Nacional de Costa Rica. Revista Universidad en Diálogo, 7(2), 37-71. Recuperado de https://bit.ly/38dOFDL

\section{BY-NC-ND}

